\title{
Cálculo retrospectivo del coeficiente de balasto horizontal a partir de mediciones de desplazamientos en pantallas del Metro de Sevilla
}

\section{Back analysis of the coefficient of horizontal subgrade reaction from the monitored behaviour of diaphragm walls of Seville's Underground}

$\underline{\text { P. Arozamena }}^{(*)}$, A. Jaramillo ${ }^{(* *)}$, C. Oteo ${ }^{(* *)}$

\section{RESUMEN}

En este artículo se presenta un ábaco con los valores del coeficiente de balasto horizontal para el cálculo de pantallas en Sevilla. Dichos valores se han extrapolado a partir del comportamiento real en obra de las pantallas, medido con inclinómetros, durante las distintas fases de excavación. Se realiza de inicio un análisis primario utilizando los programas de cálculo Plaxis, Rido y Cype y los valores del coeficiente de balasto de Chadeisson. La comparación y discusión de los resultados obtenidos nos lleva a concluir que los cálculos habituales con Rido y Cype no son fiables para predecir adecuadamente el comportamiento real de las pantallas. Por eso se realiza un análisis paramétrico, variando los valores del coeficiente de balasto, hasta dar con aquéllos que mejor reproducen la realidad medida. Los resultados alcanzados se sintetizan en forma de ábaco para que lo aprendido con la experiencia pueda ser aplicado en futuros trabajos.

Palabras clave: Coeficiente de balasto horizontal; cálculo de pantallas; muelles elasto-plásticos; Rido; Cype; inclinómetros; Metro de Sevilla.

\section{ABSTRACT}

This paper presents an abacus with the values of the coefficient of horizontal subgrade reaction for retaining wall analysis in Seville. The aforementioned values have been extrapolated from the actual behaviour on site of the retaining walls, monitored with inclinometers, during the different excavation stages. A primary analysis is carried out initially using the calculation programs Plaxis, Rido and Cype and Chadeisson values of the coefficient of horizontal subgrade reaction. The comparison and discussion of the achieved results leads us to conclude that the usual calculations with Rido and Cype are not reliable for accurately predicting the actual behaviour of the retaining walls. Due to that a parametric analysis is carried out, varying the values of the coefficient of horizontal subgrade reaction, until finding those which mach better the measured reality. The obtained results are synthesised in an abacus so that the knowledge learnt through experience can be applied in future works.

Keywords: Coefficient of horizontal subgrade reaction; retaining wall analysis; elasto-plastic springs; Rido; Cype; inclinometers; Seville's Underground.

\author{
(*) Geotecnia y Cimientos, S. A. (España). \\ ${ }^{(* *)}$ Universidad de Sevilla (España). \\ (***) Catedrático Ing. del Terreno. Asistente Honorario Univ. de Sevilla (España). \\ Persona de contacto/Corresponding author: parozamenac@hotmail.es (P.Arozamena)
}

Cómo citar este artículo/Citation: Arozamena, P., Jaramillo, A., Oteo, C. (2015). Cálculo retrospectivo del coeficiente de balasto horizontal a partir de mediciones de desplazamientos en pantallas del Metro de Sevilla. Informes de la Construcción, 67(538): eo84, doi: http://dx.doi.org/10.3989/ic.13.148.

Licencia/License: Salvo indicación contraria, todos los contenidos de la edición electrónica de Informes de la Construcción se distribuyen bajo una licencia de uso y distribución Creative Commons Reconocimiento no Comercial 3.o. España (cc-by-nc). 


\section{INTRODUCCIÓN}

Entre junio de 2003 y noviembre de 2009 se construyó la Línea 1 del Metro de Sevilla. Seis años de obras para ejecutar una infraestructura de más de 18 kilómetros que atraviesa Sevilla de oeste a este, con tramos en superficie en la periferia y tramos subterráneos en la ciudad, excavados éstos últimos entre pantallas y también con tuneladora. El seguimiento y control técnico de las obras implicó la instalación de múltiples instrumentos de auscultación y la toma de cuantiosas lecturas en éstos, para registrar el comportamiento de la infraestructura construida y del terreno y estructuras de su entorno.

La ingeniería geotécnica, o del terreno, presenta una particularidad fundamental que la distingue del resto de ingenierías, la cual es precisamente eso: «el terreno», el hecho de involucrar a materiales naturales y complejos como suelos y rocas, cuyas propiedades varían en el espacio y en el tiempo, y cuyo comportamiento no es cabalmente conocido. En el campo de la ingeniería geotécnica no basta con la sola aplicación de las matemáticas, la física, la mecánica y otras ciencias; la experiencia y la práctica se hacen imprescindibles para poder alcanzar los objetivos propuestos. Es necesario que el ingeniero geotécnico verifique sus hipótesis preliminares de cálculo de la fase de proyecto con los resultados alcanzados a posteriori durante la obra. En cumplimiento de este precepto se elabora el presente trabajo: es conocido el comportamiento real que tuvieron las pantallas durante la excavación, partiendo de este conocimiento, se establece qué valores numéricos de los parámetros del terreno reproducen dicho comportamiento. En particular, el coeficiente que quizá sea el más hermético de todos: el coeficiente de balasto.

El primero que introdujo el concepto del coeficiente de balasto en Mecánica aplicada fue Winkler (1), en su libro sobre resistencia de materiales, en el que desarrolló sus propias soluciones a un importante número de problemas de ingeniería, basándose en un sólido análisis matemático. Tras él, el primero que utilizó el concepto en una aplicación práctica fue Zimmermann (2), para calcular los esfuerzos en raíles y traviesas de ferrocarril apoyadas sobre balasto en toda su longitud. Durante las siguientes décadas la teoría se fue expandiendo para abarcar el cálculo de esfuerzos en cimentaciones flexibles, tales como zapatas continuas o losas. Hacia 1920 la teoría del coeficiente de balasto empezó a ser utilizada en casos en los que las reacciones del terreno actuaban en dirección horizontal, tales como pilotes y tablestacas.

Terzaghi (3) fijó a modo de referencia los valores del coeficiente de balasto vertical $\left(K_{30}\right)$ para el caso de placas cuadradas de un pie por un pie de sección (y también para placas de un pie de anchura y longitud ilimitada), como base para estimar los correspondientes valores para el caso de zapatas aisladas, corridas y losas $\left(K_{v}\right)$. También incluyó en su estudio los casos de pilotes y pantallas verticales, sometidos a cargas y empujes horizontales, utilizando el concepto de coeficiente de balasto horizontal $\left(K_{h}\right)$, para el cual estableció también valores numéricos. Los valores finales que recomendó para el cálculo de estas estructuras se establecían a partir de: unos valores base para dimensiones de un pie, y del diámetro o anchura del pilote, o la profundidad de empotramiento de la pantalla respectivamente. Por la misma época, Rowe (4), (5), (6) y (7) analizó y publicó los empujes en pantallas sometidas a las acciones del terreno, y en pilotes aislados sometidos a una carga horizontal en cabeza. Ambos casos en suelos sin cohesión y basándose en análisis teóricos y ensayos experimentales.

En la década de los 50 del siglo xx se produjo el despegue de la construcción de pantallas continuas de hormigón armado utilizadas para impermeabilizar, proteger y consolidar excavaciones, tal como recoge Chadeisson (8). Este ingeniero desarrolló en los años 60 y 70 un programa informático de cálculo de pantallas en el que utilizó el coeficiente de balasto y el modelo matemático de Winkler para definir el comportamiento del terreno. Basándose en su experiencia en el cálculo de pantallas, propuso un ábaco con valores numéricos del coeficiente de balasto. No se tiene conocimiento de que el ábaco fuera publicado por aquel entonces, pero su uso parece haber sido bastante generalizado entre los ingenieros franceses, Monnet (9).

En la actualidad el cálculo de pantallas se realiza con ayuda de programas informáticos comerciales. Esto viene siendo así desde la década de los 90 del siglo xx. Entre los programas de cálculo de uso más extendido entre los profesionales se encuentran: Rido (10), Cype (11) y Plaxis (12). Se han elegido estos tres para comparar entre sí los resultados que ofrecen. Los dos primeros (Rido y Cype) ejecutan cálculos de equilibrio elasto-plástico del conjunto terreno y elementos estructurales (pantalla, anclajes, arriostramientos, etc.). Los cálculos los realizan por fases, siguiendo la secuencia de ejecución de la obra, ya que ésta condiciona los esfuerzos y las deformaciones, dada la irreversibilidad del comportamiento del terreno y la variación de las cargas y la geometría de la construcción con las distintas fases. Ambos programas están basados en modelo de los muelles elasto-plásticos caracterizados por el coeficiente de balasto introducido por Haliburton (13). En éste modelo el suelo se sustituye por un conjunto de muelles horizontales independientes caracterizados por una relación presión-desplazamiento bilineal elasto-plástica. El coeficiente de balasto (rigidez del muelle) es la pendiente inicial de la recta, hasta que se alcanza la presión límite, activa o pasiva. De esta forma un problema 2-D (deformación plana) se convierte en un problema 1-D. El comportamiento del terreno se puede englobar en un único parámetro $\left(K_{h}\right)$. El caso más simple de medio lineal elástico homogéneo e isótropo requiere un mínimo de dos parámetros $(E$ y $v$, o $G$ y $K)$ para quedar completamente definido.

El tercero de los programas (Plaxis) es un programa de elementos finitos que realiza cálculos de deformación y estabilidad de una gran variedad de problemas geotécnicos, las pantallas constituyen tan solo una de las posibles aplicaciones de Plaxis. Lógicamente, también tiene en cuenta en el cálculo las fases de ejecución de la obra, y con él es posible modelizar los distintos elementos estructurales.

El programa Rido ha sido desarrollado y lo comercializa la empresa Robert Fages Logiciels, especializada en este programa de cálculos de pantallas y con sede en Miribel, situada a $14 \mathrm{~km}$ al nordeste de Lyon. Su distribución está a nivel mundial.

El programa Cype Muros Pantalla ha sido desarrollado y lo comercializa la empresa Cype Ingenieros, la cual se ha especializado en software técnico para Arquitectura, Ingeniería y Construcción. Tiene su sede en Alicante. Su distribución 
principalmente alcanza a España, Portugal y Latinoamérica, y en menor medida Norteamérica, Europa y Asia.

El programa Plaxis fue desarrollado inicialmente en Universidad Técnica de Delft en Holanda y lo comercializa la empresa Plaxis, creada con posterioridad y con sede en la misma ciudad. Su distribución está a nivel mundial.

Dado que hay una gran disparidad de valores publicados para el coeficiente de balasto la elección de su valor es una tarea particularmente difícil para los proyectistas de pantallas. El presente trabajo pretende arrojar luz en este proceso, aportando la experiencia de las obras del Metro de Sevilla. El artículo se organiza según se muestra en la Figura 1.

\section{TRABAJOS DE CAMPO}

Basándose en los registros de los inclinómetros, se establece de inicio el comportamiento real en obra de las pantallas durante las distintas fases de excavación, en tres emplazamientos clave que caracterizan el conjunto de la traza de la Línea 1 del Metro de Sevilla: las estaciones Plaza de Cuba y San Bernardo y un tramo de túnel en la calle Blas Infante.

\subsection{Estación Plaza de Cuba}

La Estación Plaza de Cuba es la más profunda de las situadas al oeste de la dársena del Guadalquivir. La excavación alcanza 20,50 m con respecto al nivel de la calle y se lleva a cabo en 6 fases. Las pantallas de hormigón armado son de 1,00 $\mathrm{m}$ de espesor y 33,00 $\mathrm{m}$ de profundidad.

El comportamiento real en obra de las pantallas durante las distintas fases de excavación, está representado mediante las líneas continuas de la Figura 2.

\subsection{Estación San Bernardo}

La Estación San Bernardo está situada al este de la dársena del Guadalquivir. Su profundidad de excavación alcanza 20,50 m de profundidad con respecto al nivel de la calle y se lleva a cabo en 4 fases. Las pantallas de hormigón armado son de 1,00 $\mathrm{m}$ de espesor y 32,00 $\mathrm{m}$ de profundidad.
El comportamiento real en obra de las pantallas durante las distintas fases de excavación, está representado mediante las líneas continuas de la Figura 3.

\subsection{Túnel de Blas Infante}

El túnel de Blas Infante constituye el inicio de la parte subterránea de la Línea 1 del Metro, en la zona oeste de la ciudad de Sevilla. La excavación de la rampa se realiza en voladizo y alcanza 7,50 m de profundidad en 6 fases. Las pantallas de hormigón armado son de 0,80 m de espesor y 20,00 m de profundidad.

El comportamiento real en obra de las pantallas durante las distintas fases de excavación, está representado mediante las líneas continuas de la Figura 4.

\section{ANÁLISIS PRIMARIO}

A modo de análisis primario se realizan varios cálculos en cada uno de los tres emplazamientos mencionados, utilizando los tres programas elegidos. Para cubrir todo el espectro se analizan tres hipótesis sobre la magnitud del rozamiento terreno-pantalla, la cual condiciona la inclinación de los empujes activo y pasivo: $\delta=0, \delta=\varphi / 3$ y $\delta=2^{*} \varphi / 3$. El corte estratigráfico y los valores de los parámetros geomecánicos de los distintos niveles de terreno en cada emplazamiento se obtienen a partir de los resultados de los sondeos mecánicos a rotación y de los ensayos de laboratorio ejecutados a lo largo de la traza por diferentes empresas, y recogidos en el proyecto de construcción. Éstos se resumen en las Tablas 1, 2 y 3 . El emplazamiento del casco actual de Sevilla (vega del Río Guadalquivir) históricamente fue zona inundable (los romanos vivían en la zona alta de Santiponce). El emplazamiento fue rellenado poco a poco durante el transcurso de la Edad Media de forma que, ahora, el nivel de rellenos antrópicos tiene unos 2-5 $\mathrm{m}$ de espesor, dependiendo de la zona. En el centro de la ciudad, bajo los rellenos antrópicos se presentan depósitos cuaternarios constituidos por capas de arenas flojas y arcillas blandas a medias. La cohesión efectiva media de la fracción plástica de las arcillas arenosas es $11 \mathrm{kPa}$ y el ángulo de rozamiento efectivo medio $27,5^{\circ}$. Por lo que respecta a la fracción no plástica de las arcillas arenosas,

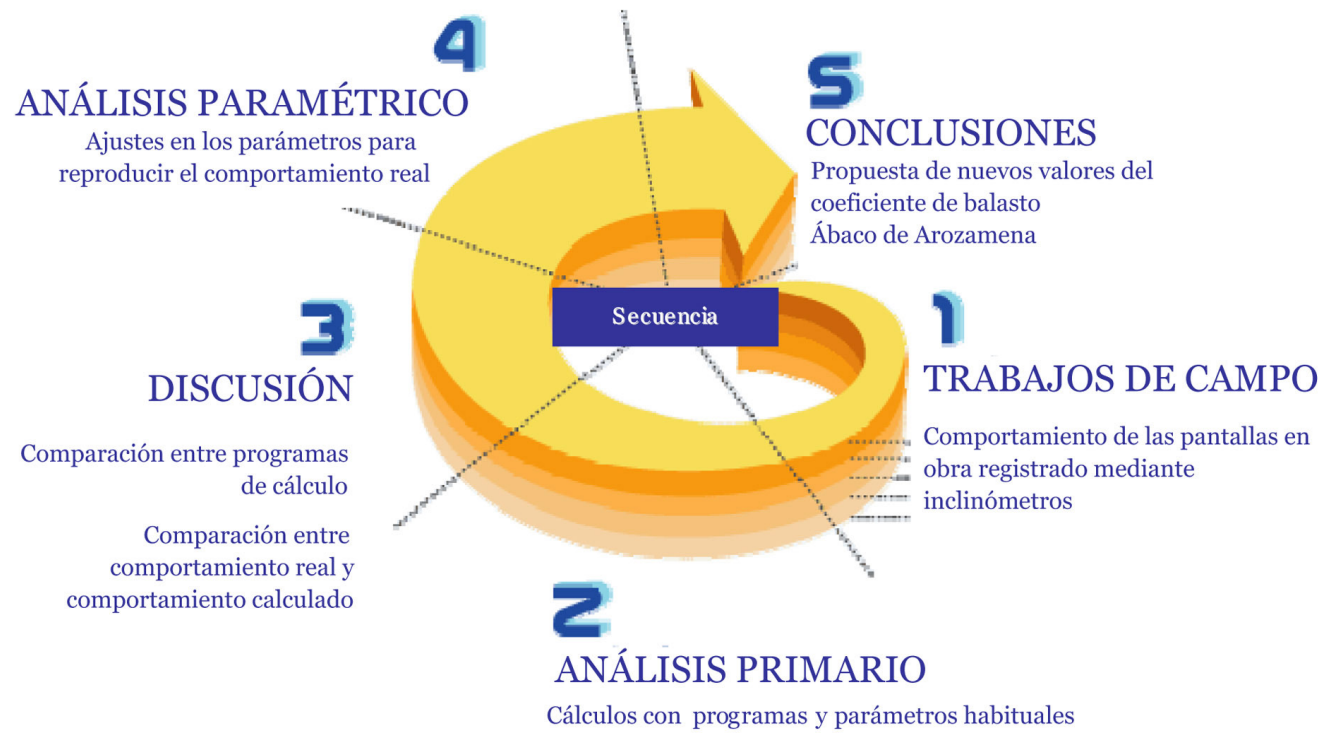

Figura 1. Secuencia de trabajos realizados. 
su cohesión efectiva media es nula y su ángulo de rozamiento efectivo medio $35^{\circ}$. Por debajo, las gravas cuaternarias se presentan en todo el ámbito de la vega del Río Guadalquivir. Suelen tener tamaños que varían entre 20 y $80 \mathrm{~mm}$, con algunas capas arenosas intercaladas. Generalmente los valores del SPT en las gravas varían entre 25 golpes cada $30 \mathrm{~cm}$ y rechazo. La cohesión efectiva media de las gravas es nula y el ángulo de rozamiento efectivo medio $35^{-45^{\circ}}$. El sustrato está constituido por las denominadas «Margas Azules del Guadalquivir», que ni son margas ni son azules. Se trata de arcillas miocenas fisuradas, de plasticidad media a alta (límite líquido de 40 a 70), algo expansivas (sobre todo en superficie), con resistencia a compresión simple variable entre 0,4 y 1,2 MPa en la ciudad. La cohesión efectiva media de la fracción plástica de las arcillas arenosas es $12-30 \mathrm{kPa}$ y el ángulo de rozamiento efectivo medio $25-34^{\circ}$. Los valores de los parámetros geotécnicos están recogidos en Oteo (14). El nivel freático viene influido principalmente por la cota del río, la dársena y la topografía de la ciudad y se encuentra entre las profundidades 3 y $8 \mathrm{~m}$.

Como ya se ha mencionado en la introducción, existe una gran disparidad de valores publicados del coeficiente de balasto horizontal para pantallas. Incluso hay discrepancias entre los diversos autores al establecer qué factores influyen en el coeficiente y cómo influyen éstos.

Terzaghi (3) establece que el coeficiente depende de las propiedades elásticas del terreno y de las dimensiones del área cargada, con un valor constante para arcillas (dependiendo de su consistencia) y con valores crecientes linealmente con la profundidad para arenas (dependiendo de su compacidad).
Monnet (9) atribuye a Chadeisson un ábaco en el que $K_{h}$ depende exclusivamente del terreno, con valores en función de la cohesión y del ángulo de rozamiento. Éstos son constantes, tanto para arcillas como para arenas.

Monnet (9), en su fórmula, reproduce razonablemente los valores del ábaco de Chadeisson, e introduce como factor adicional la rigidez de la pantalla. El valor del coeficiente de balasto sube cuanto mayor sea la rigidez de la pantalla, especialmente para suelos arenosos.

Schmitt (15) hace depender el coeficiente de balasto de las características del terreno y de la geometría y rigidez de la pantalla. La particularidad es que, al contrario de lo concluido por Monnet, el valor del coeficiente de balasto baja cuanto mayor sea la rigidez de la pantalla, tanto para arcillas como para arenas.

Bowles (16) presenta una relación entre $E$ y $K_{v}$ a partir de la teoría de la elasticidad definida por Vesic (17) y (18) y recomienda no utilizar las expresiones de Terzaghi por carecer de base teórica.

Muzás (19) expone que los valores del coeficiente de balasto horizontal para pantallas de Terzaghi son conservadores. También concluye que para suelos intermedios el coeficiente de balasto a considerar debe ser de tipo trapecial, como combinación de los criterios de arcilla y arena.

Monaco y Marchetti (20) establecen que $K_{h}$ depende, no solo de la rigidez del suelo, sino también de la profundidad de excavación y la altura entre arriostramientos de la pantalla. En

Tabla 1. Corte estratigráfico en la Estación Plaza de Cuba.

\begin{tabular}{|l|c|c|c|c|c|c|c|}
\hline Nivel & $\begin{array}{c}\text { Prof. de techo } \\
(\mathbf{m})\end{array}$ & $\begin{array}{c}\text { Prof. de muro } \\
(\mathbf{m})\end{array}$ & $\begin{array}{c}\text { Peso específico } \\
\left(\mathbf{k N} / \mathbf{m}^{\mathbf{3}}\right)\end{array}$ & $\begin{array}{c}\text { Cohesión } \\
(\mathbf{k P a})\end{array}$ & $\begin{array}{c}\text { Ángulo de } \\
\text { rozamiento }\end{array}$ & $\begin{array}{c}\text { Módulo de } \\
\text { deformación } \\
\boldsymbol{E} \mathbf{( M P a )}\end{array}$ & $\begin{array}{c}\text { Coeficiente de } \\
\text { balasto Chadeisson } \\
\boldsymbol{K}_{\boldsymbol{h}}\left(\mathbf{k N} / \mathbf{m}^{3}\right)\end{array}$ \\
\hline Relleno & 0,00 & 2,00 & 19 & 10 & $25^{\circ}$ & 10 & 22.000 \\
\hline Arena & 2,00 & 15,00 & 21 & 5 & $34^{\circ}$ & 24 & 40.000 \\
\hline Grava & 15,00 & 23,00 & 21 & 0 & $37^{\circ}$ & 42 & 46.000 \\
\hline Marga & 23,00 & $>50,00$ & 20 & 40 & $28^{\circ}$ & 52 & 35.000 \\
\hline
\end{tabular}

Tabla 2. Corte estratigráfico en la Estación San Bernardo.

\begin{tabular}{|l|c|c|c|c|c|c|c|}
\hline Nivel & $\begin{array}{c}\text { Prof. de techo } \\
(\mathbf{m})\end{array}$ & $\begin{array}{c}\text { Prof. de muro } \\
\mathbf{( m )}\end{array}$ & $\begin{array}{c}\text { Peso específico } \\
\left(\mathbf{k N} / \mathbf{m}^{\mathbf{3}}\right)\end{array}$ & $\begin{array}{c}\text { Cohesión } \\
\mathbf{( k P a )}\end{array}$ & $\begin{array}{c}\text { Ángulo de } \\
\text { rozamiento }\end{array}$ & $\begin{array}{c}\text { Módulo de } \\
\text { deformación } \\
\boldsymbol{E}(\mathbf{M P a})\end{array}$ & $\begin{array}{c}\text { Coeficiente de } \\
\text { balasto Chadeisson } \\
\boldsymbol{K}_{\boldsymbol{h}}\left(\mathbf{k N} / \mathbf{m}^{3}\right)\end{array}$ \\
\hline Relleno & 0,00 & 1,00 & 19 & 10 & $25^{\circ}$ & 10 & 22.000 \\
\hline Arcilla & 1,00 & 10,00 & 20 & 15 & $28^{\circ}$ & 19 & 28.000 \\
\hline Grava & 10,00 & 19,50 & 21 & 0 & $37^{\circ}$ & 42 & 46.000 \\
\hline Marga & 19,50 & $>50,00$ & 20 & 40 & $28^{\circ}$ & 52 & 35.000 \\
\hline
\end{tabular}

Tabla 3. Corte estratigráfico en el Túnel de Blas Infante.

\begin{tabular}{|l|c|c|c|c|c|c|c|}
\hline Nivel & $\begin{array}{c}\text { Prof. de techo } \\
(\mathbf{m})\end{array}$ & $\begin{array}{c}\text { Prof. de muro } \\
\mathbf{( m )}\end{array}$ & $\begin{array}{c}\text { Peso específico } \\
\left(\mathbf{k N} / \mathbf{m}^{\mathbf{3}}\right)\end{array}$ & $\begin{array}{c}\text { Cohesión } \\
\mathbf{( k P a )}\end{array}$ & $\begin{array}{c}\text { Ángulo de } \\
\text { rozamiento }\end{array}$ & $\begin{array}{c}\text { Módulo de } \\
\text { deformación } \\
\boldsymbol{E}(\mathbf{M P a})\end{array}$ & $\begin{array}{c}\text { Coeficiente de } \\
\text { balasto Chadeisson } \\
\boldsymbol{K}_{\boldsymbol{h}}\left(\mathbf{k N} / \mathbf{m}^{3}\right)\end{array}$ \\
\hline Relleno & 0,00 & 3,00 & 19 & 10 & $25^{\circ}$ & 10 & 22.000 \\
\hline Arena & 3,00 & 17,00 & 21 & 5 & $34^{\circ}$ & 24 & 40.000 \\
\hline Grava & 17,00 & 21,00 & 21 & 0 & $37^{\circ}$ & 42 & 46.000 \\
\hline Marga & 21,00 & $>50,00$ & 20 & 40 & $28^{\circ}$ & 52 & 35.000 \\
\hline
\end{tabular}


arenas su valor tiende a permanecer constante tras alcanzarse una determinada profundidad de excavación. Para ellos la rigidez de la pantalla no influye mucho en el coeficiente de balasto.

Desde finales del siglo xx hay varios autores que se plantean el objetivo de ajustar mejor el comportamiento real de las pantallas, utilizando coeficientes de balasto diferentes entre activos y pasivos, distintos por zonas según la existencia o no de un arriostramiento o un anclaje pretensado próximos, y también distintos en función de si el nivel de tensiones es inferior o superior al máximo nivel alcanzado anteriormente.

Así, Balay (21) adapta la formulación de Ménard et al. (22) y (23) para evaluar $K_{h}$ en la totalidad de la longitud de una pantalla, adoptando un valor por encima, y otro diferente por debajo de la profundidad de excavación.

Simon (24) extiende la formulación de Ménard et al., adaptada por Balay, diferenciando $K_{h}$ por zonas. Por un lado están las zonas denominadas como de deformación libre (altura libre y longitud empotrada de una pantalla en voladizo). Y por otro lado las zonas denominadas como de deformación restringida (altura entre dos arriostramientos o anclajes y detrás de un anclaje pretensado).

Becci y Nova (25) tienen en cuenta en su método un comportamiento no lineal del suelo. Calculan $K_{h}$ a partir del módulo $E_{u r}$ cuando el nivel de tensiones es inferior al nivel máximo de tensiones, $\mathrm{y}$ a partir de $\mathrm{E}_{\text {oed }}$ cuando el nivel de tensiones supera el máximo pasado.

Muzás (26) propone la utilización de tres coeficientes de balasto en el trasdós de la pantalla y dos coeficientes en el intradós. Los del trasdós serían: uno de descarga desde el reposo hasta los empujes activos, y dos de recarga, desde el empuje activo y desde el empuje al reposo inicial. Y los del intradós serían: uno de recarga desde el empuje activo y otro de carga desde el reposo inicial hasta los empujes pasivos.

Con todas las anteriores consideraciones en mente, y teniendo en cuenta también que el uso de los valores de Chadeisson está muy extendido entre los proyectistas, se decide tomar éstos como punto de partida del análisis primario. Un extracto de los resultados alcanzados en dicho análisis primario se reproduce en las Figuras 2, 3 y 4 (líneas de puntos). Resultados que se comentan con detalle en el siguiente apartado de discusión.

\section{DISCUSIÓN}

La comparación entre los resultados alcanzados utilizando el programa Rido y los del programa Cype nos permite concluir en primer lugar que ambos programas predicen un comportamiento prácticamente igual para las pantallas. No hay diferencia en los resultados por la elección de un programa u otro.

En segundo lugar, la comparación entre los resultados obtenidos en los cálculos utilizando Plaxis, con la realidad registrada en obra mediante instrumentación, muestra que los cálculos son fiables para predecir el comportamiento de las pantallas siempre que se utilice el modelo Hardening Soil para caracterizar los terrenos y valores de $\delta$ en el entorno de $\varphi / 3-2^{*} \varphi / 3$.

En tercer lugar, la comparación entre los resultados obtenidos en los cálculos utilizando Rido y Cype, con la instrumen- tación, muestra que los cálculos (con $K_{h}$ de Chadeisson) no son fiables para predecir el comportamiento de las pantallas.

En las Figuras 2, 3 y 4 se puede observar como en la profundidad excavada de las pantallas todas las líneas de puntos (calculadas) están claramente a la izquierda de las líneas continuas (medidas en obra). Esto significa que sistemáticamente los cálculos están prediciendo movimientos inferiores a los que luego se dan en la realidad. Aquéllos son tan solo un 20-40 \% de los registrados mediante los inclinómetros. Para excavaciones de gran profundidad los cálculos se acercan más a la magnitud del desplazamiento máximo real (dependiendo del valor de $\delta$ ) pero siguen subestimando el desplazamiento en las profundidades pequeñas (cerca de donde pueden estar las cimentaciones de los edificios colindantes).

A continuación se comparan los comportamientos calculado y real en cada uno de los emplazamientos.

\subsection{Estación Plaza de Cuba}

Los desplazamientos comparados durante las distintas fases de excavación se representan en la Figura 2.

Fase 1. El desplazamiento calculado es inferior al real, aunque ambos son de pequeña magnitud. El movimiento máximo se registra en cabeza. El predicho por los cálculos es 0,3 $\mathrm{mm}$ y el medido en obra o,8 mm, para una excavación de 1,50 m.

Fase 2. El desplazamiento calculado es inferior al real y entre ellos aumenta ligeramente el factor de casi 3 que había en la fase

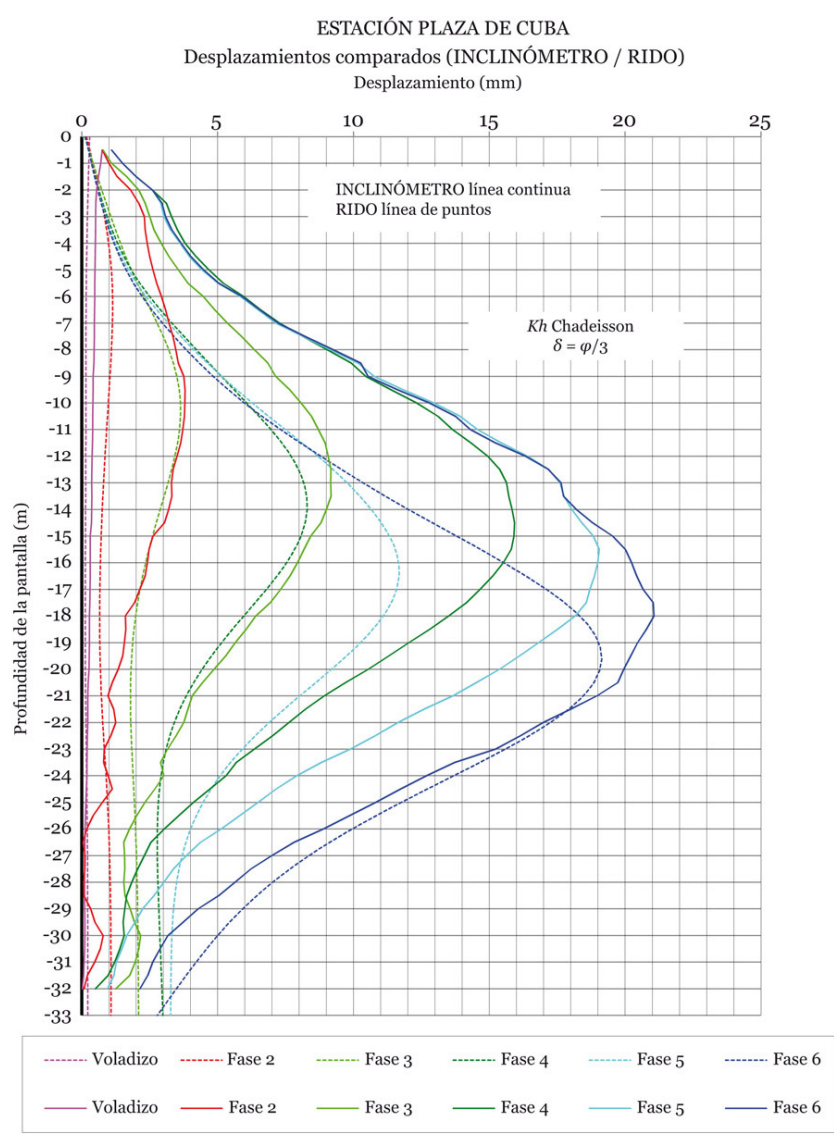

Figura 2. Estación Plaza de Cuba. Desplazamientos comparados Inclinómetro / Rido. 
anterior. El desplazamiento máximo predicho por los cálculos es 1,1 mm y el registrado 3,8 $\mathrm{mm}$ para una excavación de 6,00 $\mathrm{m}$.

Fase 3. El desplazamiento calculado continúa siendo inferior al real, aunque el factor proporcional se reduce por debajo de 3. El desplazamiento máximo predicho es $3,6 \mathrm{~mm}$ y el medido 9,2 mm, para una excavación de 10,50 m.

Fase 4. El desplazamiento calculado continúa siendo inferior al real, se incrementa algo la diferencia que había entre ellos en la fase anterior. El desplazamiento máximo calculado es $8,3 \mathrm{~mm}$ y el registrado 15,9 mm, para una excavación de 14,50 m.

Fase 5. La diferencia entre desplazamiento calculado y real se mantiene en esta fase. El desplazamiento máximo que se predice es $11,7 \mathrm{~mm}$, y el que se mide $19,0 \mathrm{~mm}$, para una excavación de 17,00 m.

Fase 6. El desplazamiento calculado $(18,9 \mathrm{~mm})$ crece considerablemente en esta fase, aunque continúa siendo inferior al real (21,1 mm). Ambos totales se igualan mucho: los cálculos subestiman los movimientos en las primeras fases y los sobrestiman en la última.

\subsection{Estación San Bernardo}

Los desplazamientos comparados durante las distintas fases de excavación se representan en la Figura 3.

Fase 1. El desplazamiento calculado es inferior al real, aunque ambos son de pequeña magnitud. El movimiento máximo se registra en cabeza. El predicho por los cálculos es $0,5 \mathrm{~mm}$ y el medido en obra 2,0 mm, para una excavación de 2,00 m.

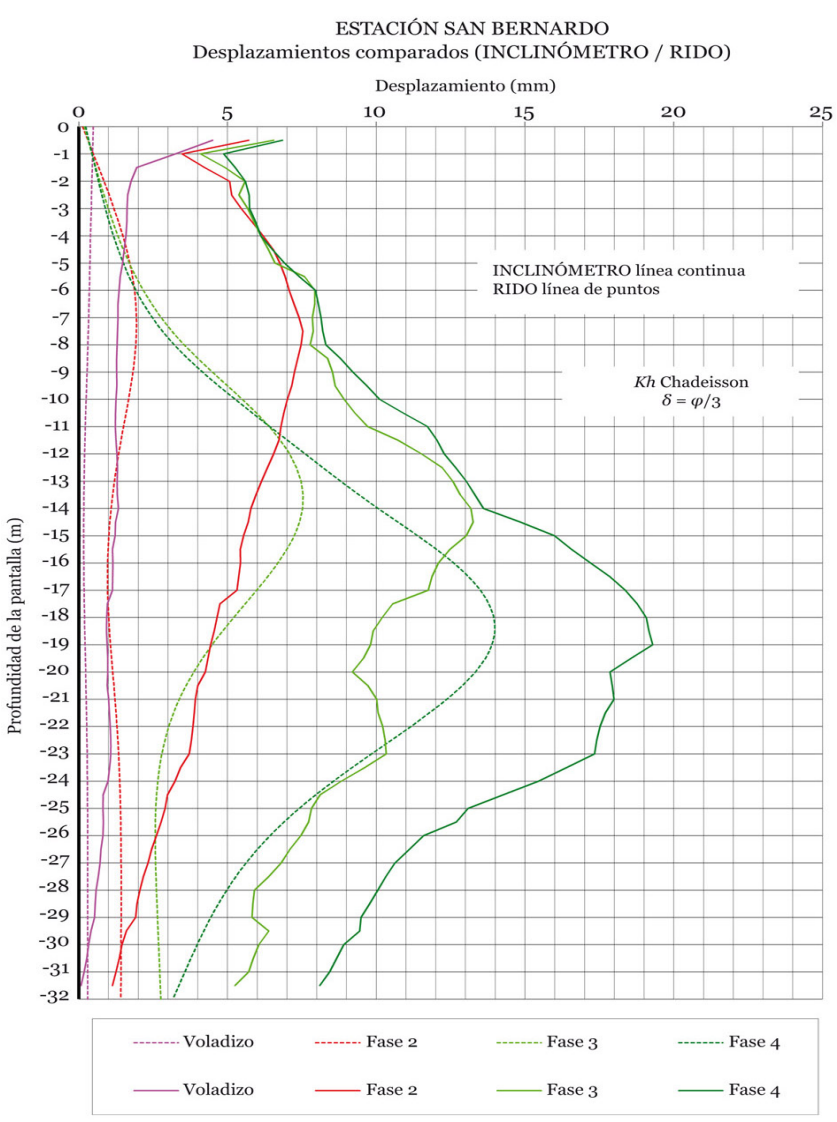

Figura 3. Estación San Bernardo. Desplazamientos comparados Inclinómetro / Rido.
Fase 2. El desplazamiento calculado es inferior al real y entre ellos se mantiene el factor de 4 que había en la fase anterior. El desplazamiento máximo calculado es $1,9 \mathrm{~mm}$ y el registrado 7,5 mm, para una excavación de 8,50 m.

Fase 3. El desplazamiento calculado continúa siendo inferior al real, prácticamente manteniéndose la diferencia de la fase anterior. El desplazamiento máximo previsto es 7,5 mm y el medido $13,3 \mathrm{~mm}$, para una excavación de $14,50 \mathrm{~m}$.

Fase 4. El desplazamiento calculado continúa siendo inferior al real, disminuyendo ligeramente la diferencia de la fase anterior. La excavación alcanza una profundidad de 20,50 m y el desplazamiento máximo previsto sube hasta 14,0 mm, mientras que el registrado sube a 19,3 $\mathrm{mm}$.

\subsection{Túnel de Blas Infante}

Los desplazamientos comparados se representan en la Figura 4. Evaluando el conjunto de fases se aprecia que el perfil general de las deformadas registradas en obra no está señalando una situación de equilibrio de la pantalla. Son demasiado verticales, la pantalla está sujeta en cabeza en cierto grado. No marcan una deformada final, sino una que va a evolucionar con el paso del tiempo. La excavación de la fase siguiente se ha realizado antes de que la deformación llegara a alcanzar su máximo para esa fase.

Fase 1. Para una excavación en voladizo de 3,50 m el movimiento máximo en cabeza de la pantalla se calcula en $1,0 \mathrm{~mm}$ y se registran $0,3 \mathrm{~mm}$. A la profundidad máxima de excavación el movimiento previsto es o,6 mm y el medido o,4 mm.

Fase 2. Para una excavación en voladizo de 4,20 m el movimiento máximo en cabeza de la pantalla se calcula en $1,3 \mathrm{~mm}$ y se registran $0,6 \mathrm{~mm}$. A la profundidad máxima de excavación el movimiento previsto es $0,7 \mathrm{~mm}$ y el medido $0,9 \mathrm{~mm}$.

Fase 3. Para una excavación en voladizo de 4,90 m el movimiento máximo en cabeza de la pantalla se calcula en $2,3 \mathrm{~mm}$ y se registran $2,4 \mathrm{~mm}$. A la profundidad máxima de excavación el movimiento previsto es $1,1 \mathrm{~mm}$ y el medido $2,8 \mathrm{~mm}$.

Fase 4. Para una excavación en voladizo de 5,60 m el movimiento máximo en cabeza de la pantalla se calcula en 4,6 $\mathrm{mm}$ y se registran 3,4 $\mathrm{mm}$. A la profundidad máxima de excavación el movimiento previsto es $1,8 \mathrm{~mm}$ y el medido 4,o mm.

Fase 5. Para una excavación en voladizo de 6,30 $\mathrm{m}$ el movimiento máximo en cabeza de la pantalla se calcula en 9,0 $\mathrm{mm}$ y se registran 4,3 $\mathrm{mm}$. A la profundidad máxima de excavación el movimiento previsto es $3,2 \mathrm{~mm}$ y el medido $4,5 \mathrm{~mm}$.

Fase 6. Para una excavación en voladizo de 7,00 m el movimiento máximo en cabeza de la pantalla se calcula en $16,9 \mathrm{~mm}$ y se registran 12,0 $\mathrm{mm}$. A la profundidad máxima de excavación el movimiento previsto es $5,5 \mathrm{~mm}$ y el medido 7,1 mm. El desplazamiento previsto por los cálculos en cabeza supera al registrado, mientras que el predicho para la excavación máxima es inferior al medido. 


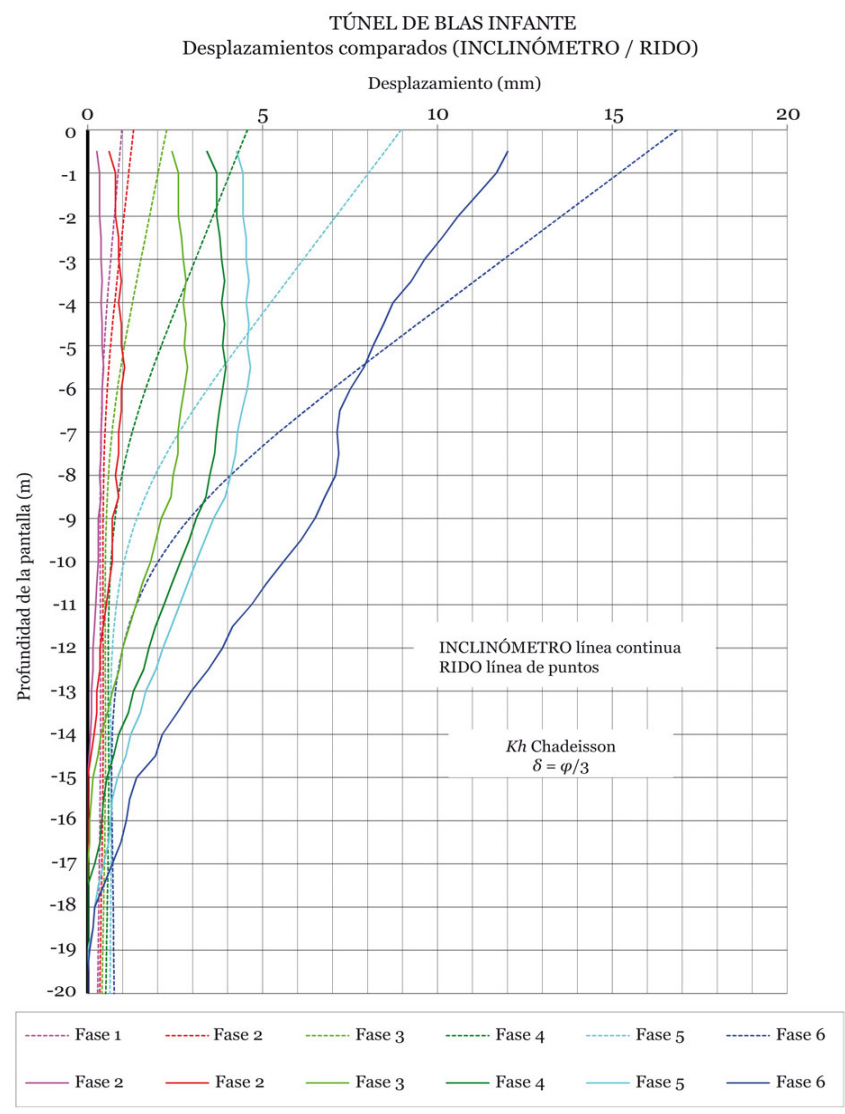

Figura 4. Túnel de Blas Infante. Desplazamientos comparados Inclinómetro / Rido.

\section{ANÁLISIS PARAMÉTRICO}

En la discusión anterior se ha establecido que los cálculos realizados con Rido y Cype como análisis primario no se asemejan lo suficiente al comportamiento real registrado en obra. Por este motivo se realiza un análisis paramétrico, basado en una nueva serie de cálculos iterativos, elaborados variando el coeficiente de balasto de los distintos terrenos implicados, hasta dar con los valores de éste que mejor reproducen la realidad medida.

Los primeros metros de empotramiento en el terreno, situados inmediatamente bajo la excavación máxima de una determinada fase, son los que marcan la magnitud del desplazamiento de la pantalla. Por tanto, conociendo ésta por los inclinómetros, es posible deducir el valor numérico del coeficiente de balasto en esos metros de terreno. Además se hacen las siguientes consideraciones:

Se opta por descartar la correlación entre el coeficiente de balasto $\left(K_{h}\right)$ y el módulo de deformación del terreno $(E)$, dado que obtener la $K_{h}$ para los muelles, partiendo de la E del medio continuo implica el tratar de establecer una correlación entre parámetros de modelos diferentes, modelos que no son concordantes.

Se limita el alcance de los valores propuestos para $K_{h}$ al cálculo de pantallas de hormigón, dejando las tablestacas explícitamente fuera del alcance. De esta forma se acota el tamaño del área cargada, que en pantallas como las del Metro se Sevilla tiene una longitud bastante grande (todo el frente continuo de empuje de la pantalla, decenas de metros) y una profundidad limitada a unos pocos metros (los situados in- mediatamente por debajo de la excavación máxima de cada fase donde son máximos los valores del empuje pasivo).

Se descarta variar el valor numérico del coeficiente de balasto con la rigidez de la pantalla. No se entiende muy bien el mecanismo que los relaciona y ni siquiera hay consenso entre autores sobre si un incremento de la rigidez hace aumentar o disminuir los valores del coeficiente de balasto.

Se desestima hacer depender el valor numérico del coeficiente de balasto de las presiones efectivas verticales del terreno, y por tanto variable a medida que se va excavando en las sucesivas fases para pantallas con múltiples niveles de arriostramiento. Además de no resultar nada fácil el establecer el factor de proporcionalidad entre presiones efectivas y coeficiente de balasto, en los tanteos realizados no se han conseguido mejores ajustes que con coeficientes constantes.

Se descarta adoptar valores distintos para el coeficiente de balasto de activos y pasivos, y para el coeficiente de balasto en carga noval y en recarga. Dada la gran dispersión de valores propuestos por los diferentes autores para el coeficiente de balasto, se considera mucho más importante orientar al proyectista para que opte por el orden de magnitud correcto para un único coeficiente de balasto, que el complicar el tema eligiendo dos o más valores distintos. Especialmente teniendo en cuenta el escaso grado de conocimiento que habitualmente se tiene de los distintos suelos que hay en un determinado emplazamiento.

Se propone que el rozamiento terreno-pantalla a considerar en los cálculos esté en el rango: $\delta=\varphi / 3-2^{*} \varphi / 3$. Se recomienda descartar la opción $\delta=$ o porque el considerar rozamiento nulo entre el terreno y la pantalla hace que los programas de cálculo con muelles elasto-plásticos sobrestimen de forma irreal los movimientos para excavaciones profundas.

\subsection{Estación Plaza de Cuba}

El mejor ajuste encontrado de los resultados de los cálculos con la realidad medida en los inclinómetros se muestra en la Figura 5.

Las curvas se han podido acoplar bastante bien en todas las fases de excavación: bajan con la misma pendiente en las profundidades correspondientes a la zona excavada de las pantallas y también se amoldan en las zonas de empotramiento.

Los desplazamientos máximos de las seis fases no muestran diferencias significativas entre las curvas del inclinómetro y las de Rido. Las diferencias máximas son del $8 \%$.

La deformada de la pantalla durante las primeras fases de excavación es la que permite ajustar el valor de $K_{h}$ en el nivel de arena situado entre 2 y $15 \mathrm{~m}$ de profundidad. La deformada de la pantalla durante las últimas fases de excavación permite ajustar el valor de $K_{h}$ en los niveles de grava y marga situados a partir de $15 \mathrm{~m}$.

\subsection{Estación San Bernardo}

El mejor ajuste encontrado de los resultados de los cálculos con la realidad medida en los inclinómetros se representa en la Figura 6.

Las curvas se han podido acoplar bastante bien en todas las fases de excavación: bajan con la misma pendiente en las 


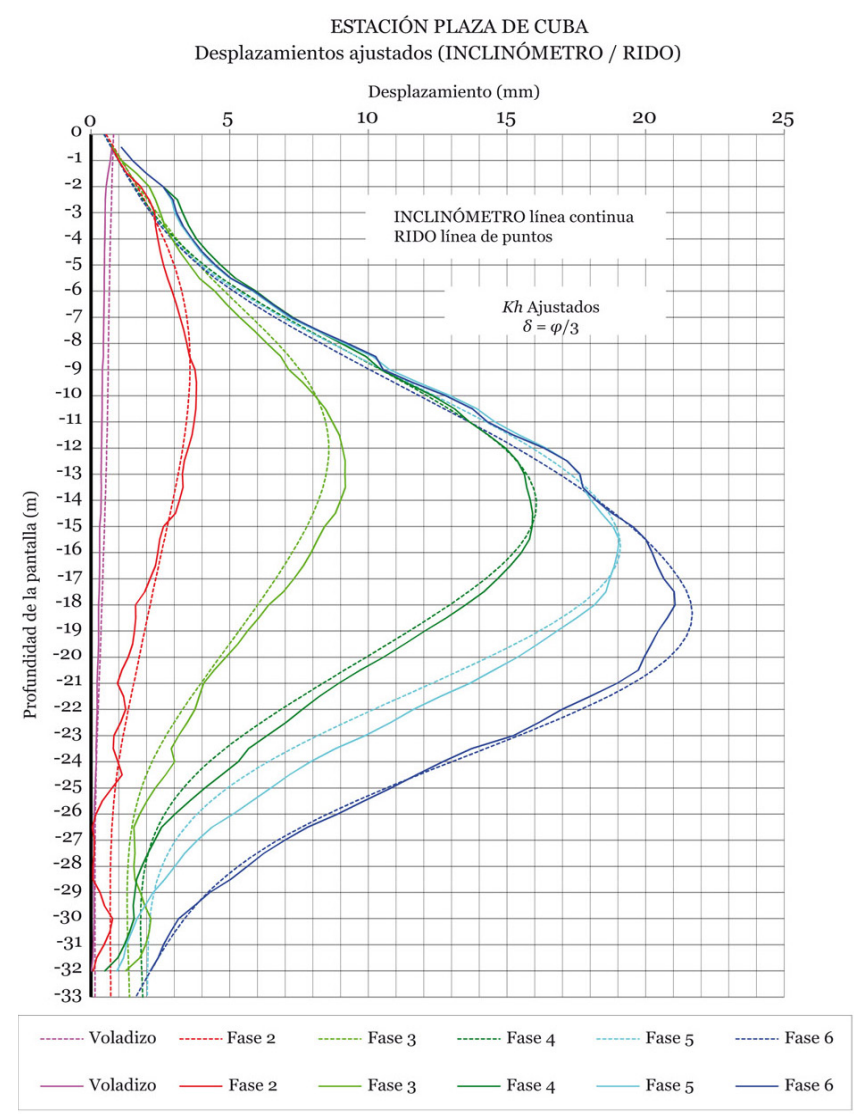

Figura 5. Estación Plaza de Cuba. Desplazamientos ajustados Inclinómetro / Rido. profundidades correspondientes a la zona excavada de las pantallas y también se amoldan en las zonas de empotramiento, teniendo en cuenta las irregularidades de las curvas del inclinómetro.

La deformada de la pantalla durante las primeras fases de excavación es la que permite ajustar el valor de $K_{h}$ en el nivel de arcilla situado entre 1 y $10 \mathrm{~m}$ de profundidad. La deformada de la pantalla durante las últimas fases de excavación permite ajustar el valor de $K_{h}$ en el nivel de grava situado entre 10,00 y 19,50 m. Los movimientos de la parte empotrada de la pantalla, y particularmente los del pie, permiten ajustar el valor del coeficiente de balasto de la marga.

\section{5•3. Túnel de Blas Infante}

El mejor ajuste encontrado de los resultados de los cálculos con la realidad medida en los inclinómetros se representa en la Figura 7.

Puede apreciarse que las curvas del inclinómetro no admiten un buen ajuste a lo largo de toda su longitud, dada su excesiva verticalidad. Se ha optado por ajustar los desplazamientos en las profundidades situadas inmediatamente por debajo de la excavación máxima (entre 8 y $14 \mathrm{~m}$ ) al ser esta la zona en la que los empujes pasivos son más altos. Ya se ha comentado que la deformada registrada con el inclinómetro no se considera final (no pasó el tiempo necesario para ello).

En el Túnel de Blas Infante la deformada de la pantalla permite ajustar el valor de $K_{h}$ en el nivel de arena situado entre 3 y $17 \mathrm{~m}$ de profundidad.

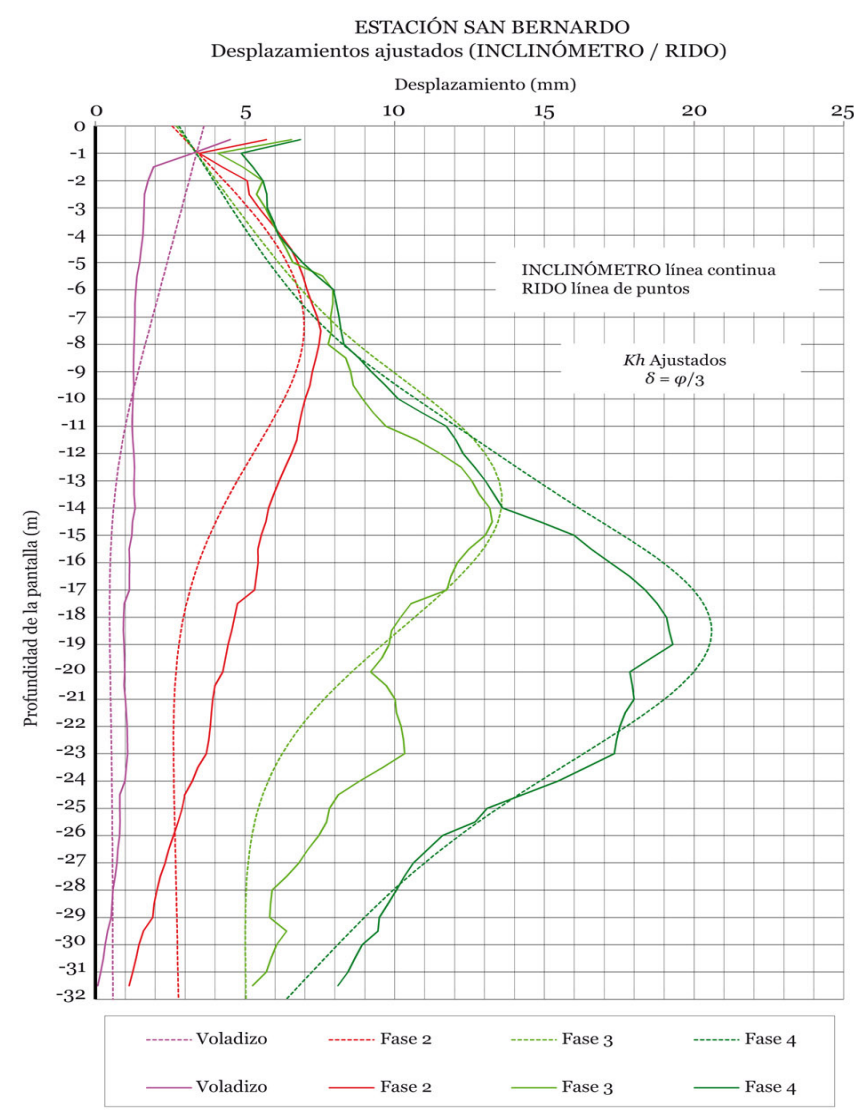

Figura 6. Estación San Bernardo. Desplazamientos ajustados Inclinómetro / Rido.

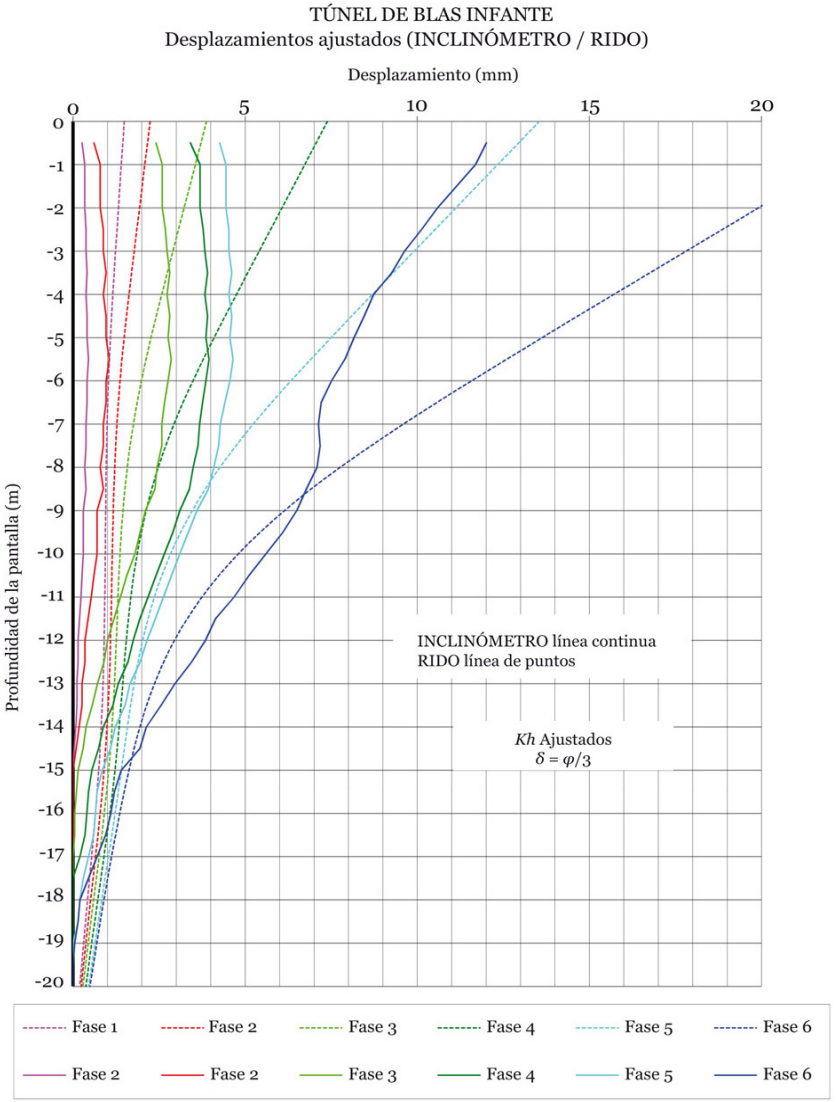

Figura 7. Túnel de Blas Infante. Desplazamientos ajustados Inclinómetro / Rido. 


\section{CONCLUSIONES}

Lo hecho en el análisis paramétrico anterior es considerar la obra del Metro de Sevilla como si fuera un conjunto de ensayos in situ a gran escala para poder determinar los valores del coeficiente de balasto horizontal para pantallas. Los suelos presentes incluyen dos tipos de suelos sin (o con poca) cohesión y dos tipos de suelos cohesivos de diferente consistencia. Éstos son lo suficientemente variados como para permitir la extrapolación de los resultados obtenidos.

Los resultados se presentan de forma clara y sencilla para que puedan tener difusión y ser utilizados, relacionando el valor numérico del coeficiente de balasto con los parámetros geomecánicos de resistencia del terreno (cohesión y ángulo de rozamiento) y adoptando el formato recogido por Monnet (9) y que él atribuye a Chadeisson.

Se propone un nuevo ábaco con valores del coeficiente de balasto horizontal para el cálculo de pantallas en los suelos de Sevilla y otros emplazamientos. Éste se reproduce en la Figura 8.

Para futuras obras de pantallas en Sevilla (del Metro u otras) se recomienda a los proyectistas la adopción de estos valores numéricos del coeficiente de balasto horizontal. El objetivo es que sus predicciones previas de movimientos se aproximen más a la realidad una vez que se realice la excavación y por tanto su evaluación de daños (con origen en esos movimientos) esté basada en resultados que mantengan su validez hasta el final de la obra.
Ábaco de Arozamena $\left(\mathrm{K}_{\mathrm{h}}\right.$ en $\left.\mathrm{kN} / \mathrm{m}^{3}\right)$

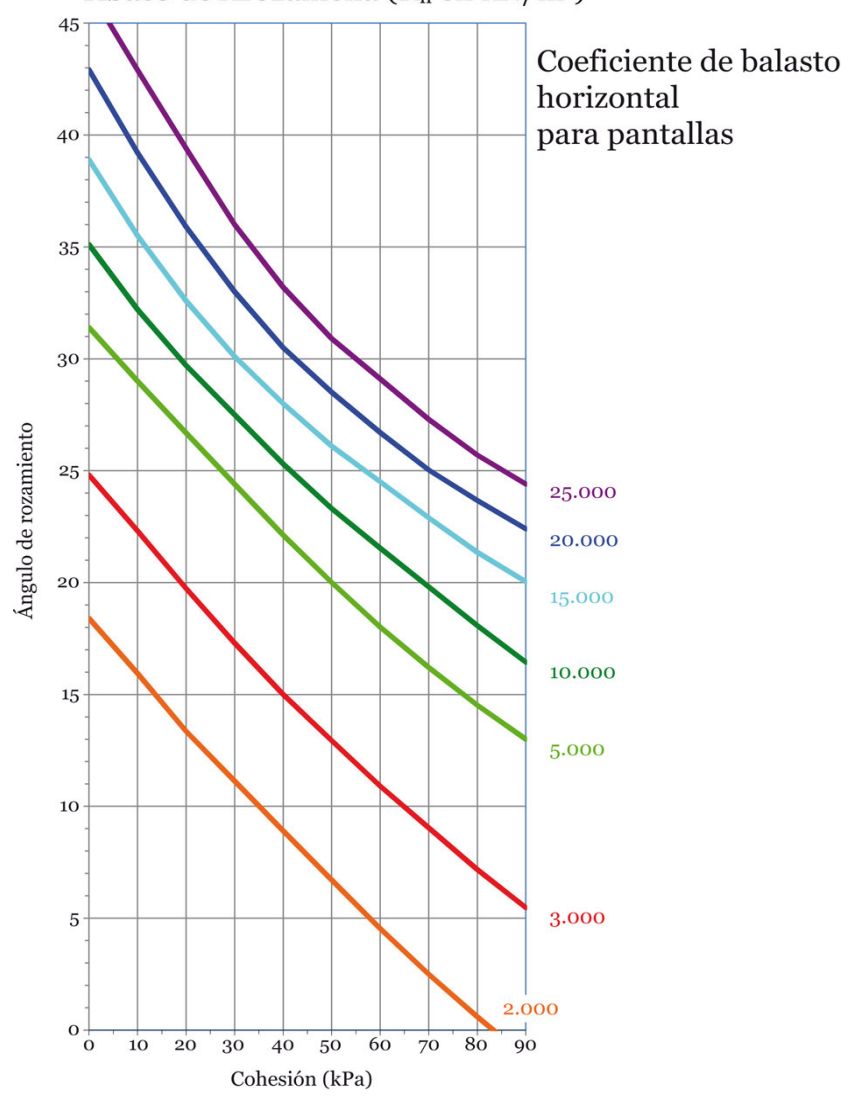

Figura 8. Ábaco de Arozamena.

\section{REFERENCIAS}

(1) Winkler, E. (1867). Die Lehre von der Elasticitaet und Festigkeit. Prague: H. Dominicus.

(2) Zimmermann, H. (1888). Die Berechnung des Eisenbahnoberbaues. Berlin: Ernst \& Korn.

(3) Terzaghi, K. (1955). Evaluation of coefficients of subgrade reaction. Geotechnique, 5(4): 297-326, doi: http://dx.doi. org/10.1680/geot.1955.5.4.297.

(4) Rowe, P.W. (1954). A stress-strain theory for cohesionless soil with applications to earth pressures at rest and moving walls. Geotechnique, 4(2): 2-70, doi: http://dx.doi.org/10.1680/geot.1954.4.2.70.

(5) Rowe, P.W. (1955). A theoretical and experimental analysis of sheet-pile walls. Proceedings of the Institution of Civil Engineers, 4(1): 1-32, doi: http://dx.doi.org/10.1680/iicep.1955.11315.

(6) Rowe, P.W. (1956a). The single pile subject to horizontal force. Geotechnique, 6(2): 70-85, doi: http://dx.doi.org/10.168o/ geot.1956.6.2.70.

(7) Rowe, P.W. (1956b). Correspondence on the article «Evaluation of coefficients of subgrade reaction» by K. Terzaghi. Geotechnique, 6(2): 94-98, doi: http://dx.doi.org/10.1680/geot.1956.6.2.94.

(8) Chadeisson, R. (1961). Parois continues moulées dans le sol. En Proceedings of the $5^{\text {th }}$ European Conference on Soil Mechanics and Foundation Engineering, 2: 563-568. Paris: Dunod.

(9) Monnet, A. (1994). Module de reaction, coefficient de décompression, au sujet des parameters utilises dans la méthode de calcul élasto-platique des soutènements. Revue Française de Géotechnique, (65): 67-72.

(10) Fages, R. (2010). WRIDO 4.12 User Manual. Miribel: Robert Fages Logiciels.

(11) Anónimo. (2007). Elementos de Contención. Muros Pantalla. Manual de Usuario. Alicante: Cype Ingenieros.

(12) Anónimo. (2002). Plaxis Manual 2D - Version 8. Lisse: A.A. Balkema Publishers.

(13) Haliburton, T.A. (1968). Numerical analysis of flexible retaining structures. Journal of Soil Mechanics \& Foundation Div, 94(SM 6 Proc Paper 6221): 1233-1251.

(14) Oteo, C. (1994). Posibles métodos constructivos para la Infraestructura. En Justo-Alpañes, J. L. (Ed.) Pasado y futuro del Metro de Sevilla, (pp. 123-151). Sevilla : Universidad de Sevilla. Colección Arquitectura no 16.

(15) Schmitt, P. (1995). Méthode empirique d'evaluation du coefficient de reaction du sol vis-á-vis des ouvrages de soutènement souples. Revue Francaise de Géotechnique, (71): 3-10.

(16) Bowles, J.E. (1996). Foundation Analysis and Design. New York : McGraw-Hill.

(17) Vesic, A.B. (1961a). Bending of beams resting on isotropic elastic solid. Journal of the Engineering Mechanics DivisionASCE, 87(2): 35-53.

(18) Vesic, A.B. (1961b). Beams on elastic subgrade and the Winkler's hypothesis. En Proceedings of the $5^{\text {th }}$ European Conference on Soil Mechanics and Foundation Engineering, 1: 845-850. Paris: Dunod. 
(19) Muzás, F. (2002). Consideraciones sobre la elección de Coeficientes de Balasto. Revista de Obras Públicas, 149(3427): 45-51.

(20) Monaco, P., Marchetti, S. (2004). Evaluation of the coefficient of subgrade reaction for design of multi-propped diaphragm walls from DTM moduli. En Proceedings of the $2^{\text {nd }}$ International Conference on Site Characterization ISC'2, 2: 993-1002. Oporto: Millpress.

(21) Balay, J. (1984). Recommandations pour le Choix des parameters de calcul des écrans de soutènement par la méthode aux modules de réaction (Note d'Information Technique). Paris : Laboratoire Central des Ponts et Chaussées.

(22) Ménard, L., Bourdon, G., Houy, A. (1964). Etude expérimentale de l'encastrement d'un rideau en fonction des caractéristiques pressiométriques du sol de fondation. Sols-Soils, (9): 11-27.

(23) Ménard, L., Bourdon, G. (1965). Calcul des rideaux de soutènement. Méthode nouvelle prenant en compte les conditions réelles d'encastrement. Sols-Soils, (12): 18-32.

(24) Simon, B. (1995). Commentaires sur le choix des coefficients de réaction pour le calcul des écrams de soutènement souples. Revue Francaise de Géotechnique, (71): 11-19.

(25) Becci, B., Nova, R. (1987). Un metodo di calcolo automatico peri l progretto di paratie. Rivista Italiana di Geotecnica, (21): 33-47.

(26) Muzás, F. (2005). El Coeficiente de Balasto en el cálculo de Pantallas. Revista de Obras Públicas, 152(3459): 33-46. 